\title{
BROADBAND SPECTRAL INVESTIGATIONS OF SGR J1550-5418 BURSTS
}

\author{
Lin Lin $^{1}$, Ersin GöĞÜşs ${ }^{1}$, Matthew G. Baring ${ }^{2}$, Jonathan Granot ${ }^{3,4,5,11}$, Chryssa Kouveliotou ${ }^{6}$, Yuki Kaneko ${ }^{1}$,

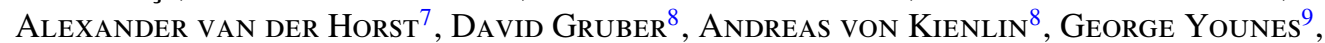 \\ AnNa L. WATts ${ }^{7}$, AND NeIl Gehrels ${ }^{10}$ \\ ${ }^{1}$ Faculty of Engineering and Natural Sciences, Sabancı University, Orhanlı Tuzla, İstanbul 34956, Turkey; linlin@ sabanciuniv.edu \\ ${ }^{2}$ Department of Physics and Astronomy, Rice University, MS-108, P.O. Box 1892, Houston, TX 77251, USA \\ ${ }^{3}$ Racah Institute of Physics, The Hebrew University, Jerusalem 91904, Israel \\ ${ }^{4}$ Raymond and Beverly Sackler School of Physics \& Astronomy, Tel Aviv University, Tel Aviv 69978, Israel \\ ${ }^{5}$ Centre for Astrophysics Research, University of Hertfordshire, College Lane, Hatfield, AL10 9AB, UK \\ ${ }^{6}$ Space Science Office, VP62, NASA/Marshall Space Flight Center, Huntsville, AL 35812, USA \\ ${ }^{7}$ Astronomical Institute "Anton Pannekoek," University of Amsterdam, Postbus 94249, 1090 GE Amsterdam, The Netherlands \\ ${ }^{8}$ Max-Planck-Institut für extraterrestrische Physik, Postfach 1312, D-85748 Garching bei Mnchen, Germany \\ ${ }^{9}$ USRA, National Space Science and Technology Center, 320 Sparkman Drive, Huntsville, AL 35805, USA \\ ${ }^{10}$ NASA Goddard Space Flight Center, Greenbelt, MD 20771, USA \\ Received 2012 March 1; accepted 2012 July 4; published 2012 August 14
}

\begin{abstract}
We present the results of our broadband spectral analysis of 42 SGR J1550-5418 bursts simultaneously detected with the Swift/X-ray Telescope (XRT) and the Fermi/Gamma-ray Burst Monitor (GBM), during the 2009 January active episode of the source. The unique spectral and temporal capabilities of the XRT windowed timing mode have allowed us to extend the GBM spectral coverage for these events down to the X-ray domain (0.5-10 keV). Our earlier analysis of the GBM data found that the SGR J1550-5418 burst spectra were described equally well with either a Comptonized model or with two blackbody functions; the two models were statistically indistinguishable. Our new broadband (0.5-200 keV) spectral fits show that, on average, the burst spectra are better described with two blackbody functions than with the Comptonized model. Thus, our joint XRT-GBM analysis clearly shows for the first time that the SGR J1550-5418 burst spectra might naturally be expected to exhibit a more truly thermalized character, such as a two-blackbody or even a multi-blackbody signal. Using the Swift and RXTE timing ephemeris for SGR J1550-5418 we construct the distribution of the XRT burst counts with spin phase and find that it is not correlated with the persistent X-ray emission pulse phase from SGR J1550-5418. These results indicate that the burst emitting sites on the neutron star need not to be co-located with hot spots emitting the bulk of the persistent X-ray emission. Finally, we show that there is a significant pulse phase dependence of the XRT burst counts, likely demonstrating that the surface magnetic field of SGR J1550-5418 is not uniform over the emission zones, since it is anticipated that regions with stronger surface magnetic field could trigger bursts more efficiently.
\end{abstract}

Key words: pulsars: individual (SGR J1550-5418, 1E 1547.0-5408, PSR J1550-5418) - stars: neutron X-rays: bursts

Online-only material: color figure

\section{INTRODUCTION}

Soft gamma repeaters (SGRs) and anomalous X-ray pulsars (AXPs) are the observational manifestations of magnetarsisolated neutron stars possessing extreme magnetic fields, $B$ $>10^{14} \mathrm{G}$ (Duncan \& Thompson 1992; Kouveliotou et al. 1998). Besides being bright X-ray sources, SGRs and AXPs emit intense bursts in hard X-rays/soft $\gamma$-rays on a highly unpredictable frequency with peak luminosities ranging from $10^{38} \mathrm{erg} \mathrm{s}^{-1}$ to $>10^{47} \mathrm{erg} \mathrm{s}^{-1}$. These energetic events are attributed to the cracking of the solid neutron star crust by magnetic stress buildup (Thompson \& Duncan 1995) or to magnetic field line reconnection (Lyutikov 2003). For detailed reviews on SGRs and AXPs, see Woods \& Thompson (2006) and Mereghetti (2008).

SGR J1550-5418 was discovered as a point source with the Einstein Observatory while searching for X-ray emission from radio emitting supernova remnants (Lamb \& Markert 1981). The source was suggested to be a magnetar candidate by the

\footnotetext{
${ }^{11}$ Current address: The Open University of Israel, 1 University Road, POB
} 808, Ra'anana 43537, Israel. similarity of its persistent X-ray spectrum to AXPs and its association with a young supernova remnant (Gelfand \& Gaensler 2007). Its magnetar nature was confirmed with the detection of radio pulsations with $P=2.096 \mathrm{~s}$ and $\dot{P}=2.318 \times 10^{-11}$, corresponding to an inferred surface dipole magnetic field strength of $2.2 \times 10^{14} \mathrm{G}$ (AXP 1E1547.0 - 5408; Camilo et al. 2007). An accurate source location was also derived from the radio image, $(\mathrm{J} 2000)$ R.A. $=15^{\mathrm{h}} 50^{\mathrm{m}} 54.11 \pm 0.01$, decl. $=$ $-54^{\circ} 18^{\prime} 23^{\prime \prime} .7 \pm 0^{\prime} .1$ (Camilo et al. 2007). X-ray pulsations at the same spin period were later found with a deeper XMM-Newton observation (Halpern et al. 2008). No bursts were detected from SGR J1550-5418 until 2008 October, when both the Swift/Burst Alert Telescope (BAT) and the Fermi/Gammaray Burst Monitor (GBM) were triggered by numerous bursts from the source (Israel et al. 2010; von Kienlin et al. 2012). SGR J1550-5418 entered an episode of more active bursting in late 2009 January, and no more bursts were detected after 2009 April. During these active episodes, several high energy instruments, such as the Swift/BAT and X-ray Telescope (XRT), the Fermi/GBM, the Rossi X-ray Timing Explorer $(R X T E) /$ Proportional Counter Array, and the International Gamma-Ray Astrophysics Laboratory (INTEGRAL)/Imager on 
board the INTEGRAL Satellite (IBIS) and the SPectrometer on INTEGRAL (SPI) recorded hundreds of bursts (Mereghetti et al. 2009; Savchenko et al. 2010; Kaneko et al. 2010; Scholz \& Kaspi 2011; van der Horst et al. 2012; von Kienlin et al. 2012). Following these burst active periods, both the persistent $\mathrm{X}$-ray emission characteristics and the spin-down behavior of the source changed remarkably (Enoto et al. 2010; $\mathrm{Ng}$ et al. 2011; Bernardini et al. 2011; Scholz \& Kaspi 2011; Dib et al. 2012; Kuiper et al. 2012).

The spectral properties of SGR J1550-5418 bursts have been extensively studied using individual instruments: SPI (Mereghetti et al. 2009), IBIS (Savchenko et al. 2010), BAT (Israel et al. 2010), XRT (Scholz \& Kaspi 2011), and GBM (van der Horst et al. 2012). The XRT data cover a relatively narrow energy range $(0.5-10 \mathrm{keV})$ of the spectrum of a typical SGR burst. Nevertheless, Scholz \& Kaspi (2011) modeled SGR J1550-5418 burst spectra using the XRT data only in the energy range of $0.5-10 \mathrm{keV}$ with a single power law and found an average photon index of $0.17 \pm 0.33$. They also reported that there is a slight anti-correlation between the photon index and the absorbed X-ray flux. BAT provides a spectral energy coverage for SGR bursts from $15 \mathrm{keV}$ to $150 \mathrm{keV}$. Israel et al. (2010) found that the spectra of BAT detected bursts can be well described by a single blackbody function with temperatures $\sim 10 \mathrm{keV}$. Finally, in van der Horst et al. (2012) we derived the spectra for a large set of SGR J1550-5418 bursts detected with GBM in 2009 January using several continuum models. We found that in a slightly broader energy range (8-200 keV), a Comptonization model or the sum of two blackbody functions (BB+BB) can fit the SGR J1550-5418 burst spectra equally well. Note that these two models were also used to describe the spectra of other magnetar bursts in a similar energy range and revealed intriguing physical insights into the burst phenomena (Feroci et al. 2004; Olive et al. 2004; Israel et al. 2008; Lin et al. 2011).

In this paper, we combine the spectral data of bursts observed simultaneously with XRT and GBM to investigate their spectral characteristics over a broader energy band (0.5-200 keV). In particular, we concentrate on the two most plausible representations of SGR burst spectra, namely the Comptonization model and the $\mathrm{BB}+\mathrm{BB}$. Focusing on bursts with data collected over broader spectral bands enhances the chance to discriminate between different spectral models. In Section 2, we describe both the XRT and GBM observations of SGR J1550-5418 bursts and the selection of their common events' sample. We present the data reduction and analysis in Section 2.3. The broadband spectral analysis results and their physical interpretation are presented in Sections 3 and 4, respectively.

\section{OBSERVATIONS AND SIMULTANEOUS BURST IDENTIFICATION}

\subsection{Observations}

Swift/XRT is an X-ray imaging spectrometer sensitive to photons in the 0.3-10 keV energy range (Burrows et al. 2005). The telescope is operated either in photon counting (PC) mode or in windowed timing (WT) mode. Both modes provide the same spectral capabilities, however, the temporal resolution of the PC mode data is about $2.5 \mathrm{~s}$, too coarse for the very short ( $\lesssim 100 \mathrm{~ms})$ SGR bursts. Therefore, we only employ here XRT observations performed in WT mode because of its $1.7 \mathrm{~ms}$ readout time well suited to the SGR burst durations. XRT monitored the source with 46 pointed observations in WT mode between 2008
Table 1

XRT Observations of SGR J1550-5418 with Simultaneous Events with GBM

\begin{tabular}{lccc}
\hline \hline Observation ID & Date & Start Time & $\begin{array}{c}\text { Exposure }^{\mathrm{a}} \\
(\mathrm{ks})\end{array}$ \\
\hline 00340573000 & 2009 Jan 22 & $02: 26: 22$ & 6.38 \\
00340573001 & 2009 Jan 22 & $09: 18: 28$ & 9.45 \\
00030956035 & 2009 Jan 30 & $17: 49: 33$ & 2.97 \\
\hline
\end{tabular}

Note. ${ }^{\mathrm{a}}$ In WT mode.

October 1 and 2009 April 30, covering burst active episodes of SGR J1550-5418. These XRT observations were densely concentrated around the most burst active period (2009 January) with a total exposure time of $\sim 175 \mathrm{ks}$. Three out of these 46 pointings (listed in Table 1) included bursts simultaneously detected with GBM, as described in detail in the next section.

The Fermi/GBM monitors the entire sky (excluding the portion occulted by the Earth) in the energy range from $8 \mathrm{keV}$ to $1 \mathrm{MeV}$ with $12 \mathrm{Na}$ I detectors and in the $0.2-40 \mathrm{MeV}$ energy band with two BGO detectors. In its trigger mode, GBM records time-tagged event (TTE) data with high temporal and spectral resolution of $2 \mu \mathrm{s}$ and 128 energy channels, respectively. The trigger readout lasts for $600 \mathrm{~s}$ (see Meegan et al. 2009 for more details of the instrument and data types). Using the same burst finding algorithm described in van der Horst et al. (2012), we searched for triggered and untriggered events from SGR J1550-5418 during the active periods of 2008 and 2009. ${ }^{12}$ In total, we identified 692 bursts out of which, 458 events had TTE data. We only used Na I detector TTE data for our spectral analyses, as typical SGR bursts are not detected above $200 \mathrm{keV}$, and last only for a fraction of a second.

\subsection{Identification of Simultaneous Events}

To identify the events observed simultaneously with GBM and XRT, we compared the times of the 458 GBM bursts with the time intervals of the XRT observations in WT mode and found 87 common bursts. Note that some SGR bursts have multi-peaked time profiles and each peak was labeled as a burst in our initial untriggered event search. We used the convention described in van der Horst et al. (2012) to determine whether multiple peaks constituted a single event - namely, we requested that the time difference between successive burst peaks was less than a quarter of the spin period of SGR J1550-5418 $(\sim 0.5 \mathrm{~s})$. As a result, we obtained 66 SGR J1550-5418 events simultaneously observed with GBM and XRT. For each burst, we plotted the burst light curves seen with GBM and XRT to determine the time interval that includes the main emission episode and is used as our spectral extraction interval (see Figure 1). We excluded nine dim bursts that had less than 40 counts in the XRT data during the burst interval, statistically not enough for spectral analysis. Additionally, we excluded five very bright bursts that saturated the high-speed science data bus of GBM (see also van der Horst et al. 2012).

We checked all common bursts in the XRT data for pileup. We regenerated the level 2 data by including photons in all grades (0-15) from level 1 data at the position of SGR J1550-5418, using the standard XRT data processing tasks in HEASOFT. Then we calculated the average readout time for each detection area. ${ }^{13}$ We found that, besides the five GBM

\footnotetext{
12 The list of GBM triggered events is available from http://gammaray.nsstc. nasa.gov/gbm/science/magnetars/magn1550triggers.html

$137 \times 1$ pixels for XRT WT mode.
} 


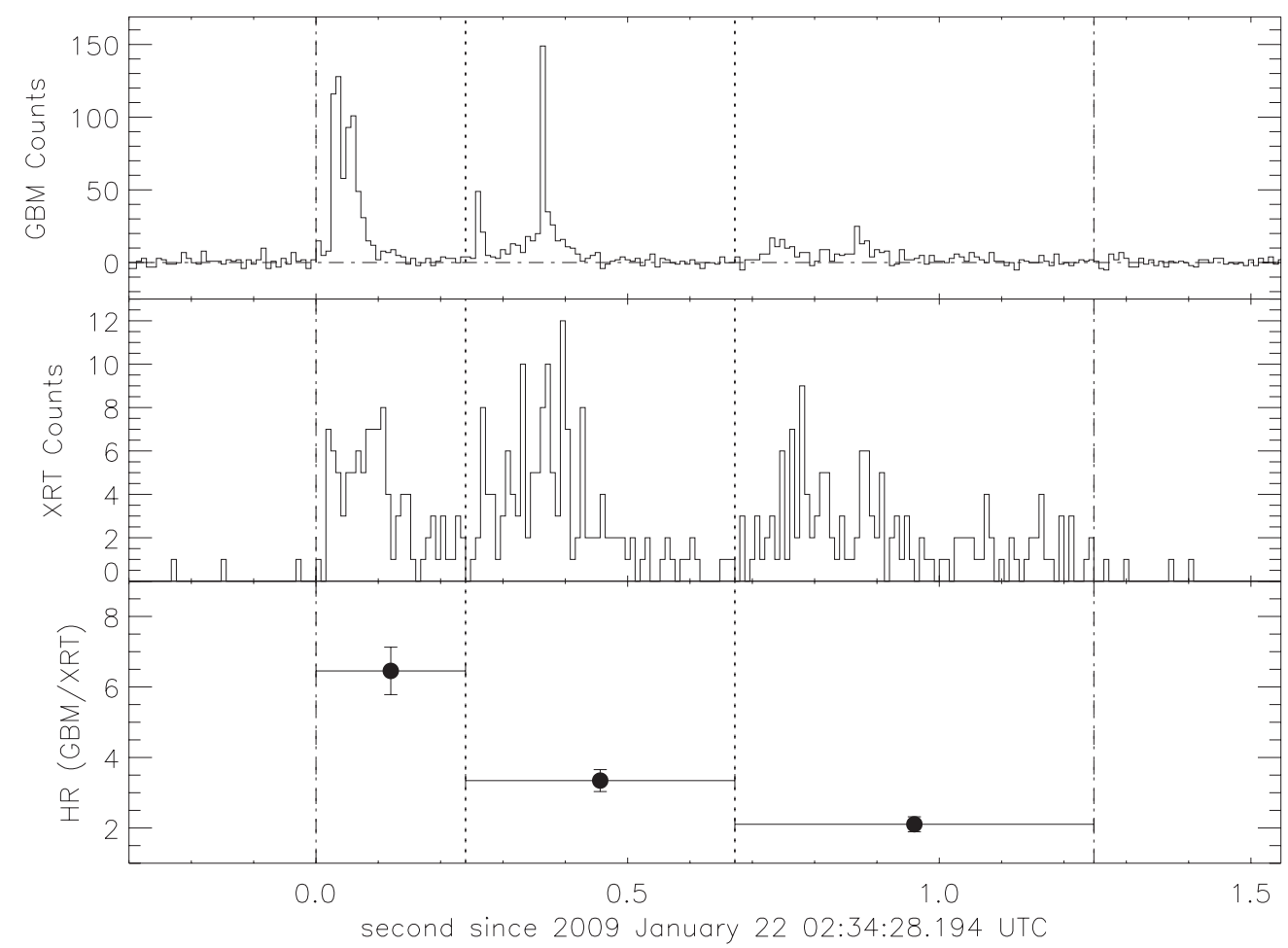

Figure 1. Top panel: background subtracted light curve of a burst detected at 02:34:28.194 on 2009 January 22 from SGR J1550-5418 with the GBM NaI-6 detector. It is binned with a time resolution of $8 \mathrm{~ms}$. Middle panel: XRT light curve of the same burst in $8 \mathrm{~ms}$ time resolution. Bottom panel: hardness ratios (GBM vs. XRT) of the three sub-intervals indicated with the dotted lines. The dot-dashed lines denote the time interval over which the burst spectrum has been accumulated.

saturated events, in ten other bursts the average readout time for central detection areas is smaller than $1.7 \mathrm{~ms}$, the smallest readout time for the WT mode. This indicates that these bursts are affected by the photon pile-up in the XRT data. Compared to the remaining common events, these bursts have more photons in grades higher than 2; as a result an analysis of the data in the good grade range (0-2) would lack most information and the results would be misleading. Therefore, we also excluded these ten piled-up bursts from further investigations. The final outcome of all these filters was a selection of 42 bursts, observed with both GBM and XRT, that we then used for broadband spectral analysis (40 bursts detected on 2009 January 22 and 2 events on 2009 January 30).

\subsection{Data Reduction}

We extracted time-integrated spectra for our 42 simultaneously detected bursts. In Figure 1, we present the burst detected at 02:34:28.194 on 2009 January 22 as an illustrative example of spectral integration ranges. We describe below the procedures we followed to generate the GBM and XRT spectra.

For the GBM data, we selected the $\mathrm{Na}$ I detectors with a source angle smaller than $60^{\circ}$, and without any blockage from the Large Area Telescope or other parts of the satellite, such as solar panels and radiators. We determined background levels by fitting pre- and post-burst intervals with a first-order polynomial using RMFIT v3.4rc12 (Mallozzi et al. 2008) and extracted both burst and background spectra. We then used grppha to group the extracted source spectrum to include at least 15 source counts in each energy bin. We generate the response matrices using GBMRSP $v 1.9$ for each burst.

For the XRT data, we selected events with grade range of $0-2$ with $x$ select, and accumulated the source spectra from a 40 pixel long section of the chip centered at the SGR J1550-5418 lo- cation in the same time intervals as used for the GBM spectra. We extracted background spectra from a region of the same size, away from the source. We generated the ancillary response function file for each burst using xrtmkarf in HEASOFT. In our spectral fitting we used the standard response file "swxwt0to2s6_20070901v012.rmf" provided in the Swift calibration database. Finally, we also grouped the source spectra to include a minimum of 15 counts in each energy bin. We fit all spectra using XSPEC v12.7.0.

\section{SPECTRAL ANALYSIS}

Motivated by our recent results published in Lin et al. (2011) and van der Horst et al. (2012), we modeled the broadband time-integrated spectra of all 42 common events with the Comptonized model (COMPT) and the sum of two blackbody functions $(\mathrm{BB}+\mathrm{BB})$. The COMPT model is in a single powerlaw shape with a high-energy exponential cutoff expressed as

$$
f=A \exp \left[-E(2+\lambda) / E_{\text {peak }}\right]\left(E / E_{\text {piv }}\right)^{\lambda},
$$

where $f$ is the photon flux in photons $\mathrm{s}^{-1} \mathrm{~cm}^{-2} \mathrm{keV}^{-1}, A$ is the amplitude with units same as $f, E_{\text {peak }}$ is the energy (in keV) at which the spectral distribution function peaks, $\lambda$ is the photon index, and $E_{\text {piv }}$ is the pivot energy fixed at $20 \mathrm{keV}$. The latter $\mathrm{BB}+\mathrm{BB}$ model has been commonly used in the context of SGR burst spectra (Feroci et al. 2004; Olive et al. 2004; Israel et al. 2008). In our spectral fits, we fix the multiplicative interstellar absorption term at $3.24 \times 10^{22} \mathrm{~cm}^{-2}$, since SGR J1550-5418 bursts are short and XRT burst spectra cannot constrain the absorption parameter. ${ }^{14}$ In our joint fits, we also include a

\footnotetext{
14 We adopt here the value obtained with the XRT observations of the persistent emission during the burst active episode of SGR J1550-5418 by Scholz \& Kaspi (2011).
} 

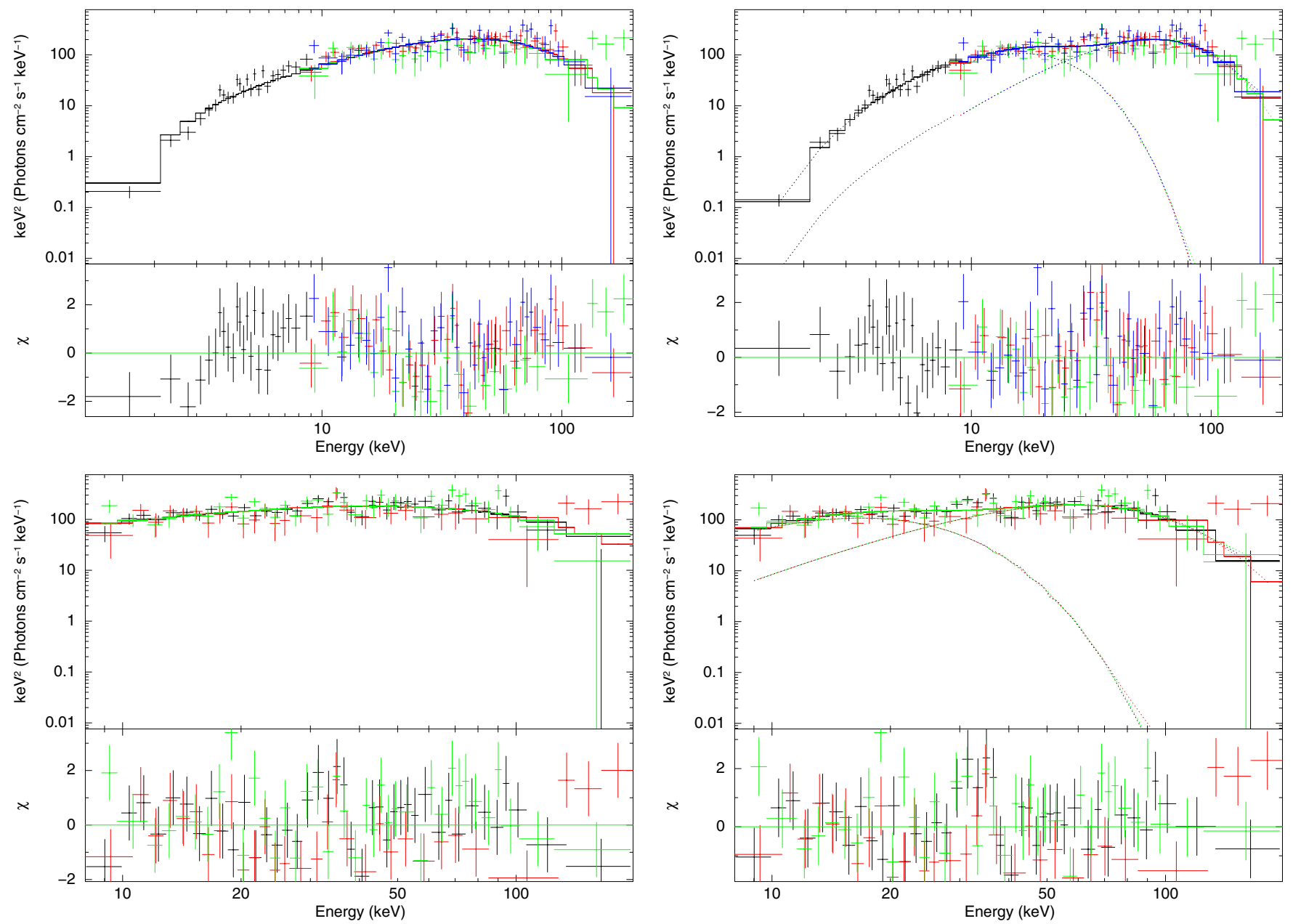

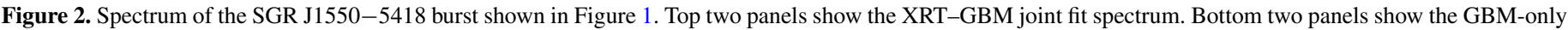
spectrum. The left column are COMPT model fits and the right column are fits with a BB+BB model. The lower parts in each panel show the fit residuals. (A color version of this figure is available in the online journal.)

multiplicative factor to account for the cross-calibration between the XRT and GBM spectra. In Figure 2, we show the broadband spectral modeling results of the burst of Figure 1 with the COMPT and BB+BB models. We note that a $\mathrm{BB}+\mathrm{BB}$ model fit to the combined XRT and GBM data results in better residuals than the COMPT ones and are also better than the COMPT fits of the GBM data alone (see the two left panels in Figure 2). These results indicate that the broadband joint fits can constrain better the physical emission model in SGR bursts as we discuss in Section 4.1. We calculated the average reduced $\chi^{2}$ for both models, and obtained 1.03 for the $\mathrm{BB}+\mathrm{BB}$ model and 1.11 for the COMPT model. The standard deviation of the reduced $\chi^{2}$ is 0.20 and 0.26 for the BB+BB and the COMPT model, respectively. These results further support the $\mathrm{BB}+\mathrm{BB}$ model against COMPT.

Table 2 presents the joint fit results with $1 \sigma$ errors for all 42 bursts. Columns 1-3 are the burst numbers, start times in UTC, and durations of the time-integrated spectra. The COMPT model parameters, i.e., the power-law index $(\lambda), E_{\text {peak }}$, and fit statistics are shown in Columns 4-6. Columns 7-13 correspond to the temperatures of the two blackbody components, the luminosity and size of the emitting area for each blackbody component (assuming a distance to SGR J1550-5418 of $5 \mathrm{kpc}$ ), and the fit statistics of the BB+BB model fits. Since the energy flux values obtained with the COMPT model are in agreement with those we get from the $\mathrm{BB}+\mathrm{BB}$ model, we only present in Columns 14 and 15 the energy flux in the GBM energy band $(8-200 \mathrm{keV})$ and the observed flux in the XRT energy band $(0.5-10 \mathrm{keV})$ using the $\mathrm{BB}+\mathrm{BB}$ fits.

The COMPT model has one less parameter than the $\mathrm{BB}+\mathrm{BB}$ function. Since these two models are not nested, we cannot employ a $\Delta \chi^{2}$ test to determine which model provides a more adequate fit. Therefore, we performed extensive simulations for each of the 42 bursts. We first fit each joint spectra with the seed model (i.e., the model with the smallest resulting $\chi^{2}$ value): these were the $\mathrm{BB}+\mathrm{BB}$ model for 33 bursts and the COMPT for the remaining nine bursts. Then, we generated 10,000 simulated spectra based on the seed model of each burst and fitted the simulated spectra with both models. For each set of 10,000 simulations, we selected fits with well-constrained model parameters, requiring the errors of $E_{\text {peak }}$ of COMPT and of the two blackbody temperatures to be less than $20 \%$. We constructed the distributions of all fit parameters as well as of the fit statistics and fitted them with a Gaussian function. The mean values of these distributions for the seed model parameters agree with the fit results listed in Table 2. Finally, we calculated the percentage of the simulated spectra which result in a smaller $\chi^{2}$ value when fitted with the seed model. This percentage, defined as the $p$-value, reflects the significance of the preference of the seed model at a given background and fluctuation level. 
Table 2

\begin{tabular}{|c|c|c|c|c|c|c|c|c|c|c|c|c|c|c|c|}
\hline No. & $\begin{array}{l}T_{\text {start }}{ }^{\mathrm{a}} \\
\text { (UTC) }\end{array}$ & $\begin{array}{l}d t \\
(\mathrm{~s})\end{array}$ & $\begin{array}{c}\text { COMPT } \\
\lambda\end{array}$ & $\begin{array}{c}\text { COMPT } \\
E_{\text {peak }} \\
(\mathrm{keV})\end{array}$ & $\begin{array}{l}\text { COMPT } \\
\chi^{2} / \text { dof }\end{array}$ & $\begin{array}{c}\text { Cool BB } \\
k T \\
(\mathrm{keV})\end{array}$ & $\begin{array}{c}\text { Hot BB } \\
k T \\
(\mathrm{keV})\end{array}$ & $\begin{array}{c}\text { Cool BB } \\
L_{39}{ }^{\mathrm{b}}\end{array}$ & $\begin{array}{c}\text { Hot BB } \\
L_{39}{ }^{b}\end{array}$ & $\begin{array}{c}\text { Cool BB } \\
R^{2} \\
\left(\mathrm{~km}^{2}\right)\end{array}$ & $\begin{array}{c}\text { Hot BB } \\
R^{2} \\
\left(\mathrm{~km}^{2}\right)\end{array}$ & $\begin{array}{l}\mathrm{BB}+\mathrm{BB} \\
\chi^{2} / \mathrm{dof}\end{array}$ & $F_{\mathrm{GBM}^{\mathrm{c}}}$ & $F_{\mathrm{XRT}}{ }^{\mathrm{d}}$ & $p$ \\
\hline 31 & $04: 34: 20.622$ & 1.184 & $-0.36_{-0.09}^{+0.10}$ & $42.43_{-1.03}^{+1.06}$ & $219.531 / 158$ & $4.62_{-0.23}^{+0.24}$ & $14.66_{-0.50}^{+0.53}$ & $1.23_{-0.07}^{+0.08}$ & $2.33_{-0.09}^{+0.09}$ & $21.13_{-3.25}^{+3.94}$ & $0.39_{-0.06}^{+0.07}$ & $165.813 / 157$ & $12.98_{-0.26}^{+0.26}$ & $1.42_{-0.08}^{+0.07}$ & $\overline{0.9998}$ \\
\hline 32 & 04:39:23.636 & 0.432 & $-0.64_{-0.23}^{+0.25}$ & $59.57_{-5.29}^{+6.60}$ & $41.121 / 50$ & $6.33_{-1.63}^{+1.52}$ & $19.46_{-3.22}^{+4.29}$ & $0.49_{-0.15}^{+0.17}$ & $0.96_{-0.16}^{+0.14}$ & $2.37_{-1.05}^{+2.08}$ & $0.05_{-0.03}^{+0.07}$ & $49.488 / 49$ & $2.01_{-0.14}^{+0.12}$ & $0.35_{-0.05}^{+0.04}$ & 0.8472 \\
\hline 33 & 04:39:29.908 & 0.184 & $-0.71_{-0.18}^{+0.19}$ & $41.63_{-2.10}^{+2.16}$ & $77.969 / 67$ & $4.07_{-0.33}^{+0.35}$ & $14.05_{-0.71}^{+0.77}$ & $1.42_{-0.14}^{+0.14}$ & $2.87_{-0.19}^{+0.18}$ & $40.24_{-10.66}^{+15.06}$ & $0.57_{-0.12}^{+0.15}$ & $64.717 / 66$ & $2.38_{-0.10}^{+0.08}$ & $0.31_{-0.04}^{+0.04}$ & 0.9585 \\
\hline 34 & $04: 39: 36.628$ & 0.336 & $-1.18_{-0.21}^{+0.23}$ & $36.93_{-3.76}^{+3.98}$ & $50.047 / 53$ & $4.16_{-0.34}^{+0.37}$ & $16.12_{-1.47}^{+1.61}$ & $0.87_{-0.08}^{+0.07}$ & $1.10_{-0.10}^{+0.10}$ & $22.80_{-6.30}^{+9.08}$ & $0.13_{-0.04}^{+0.06}$ & $35.201 / 52$ & $1.95_{-0.12}^{+0.10}$ & $0.35_{-0.05}^{+0.04}$ & 0.9943 \\
\hline 35 & 05:49:17.289 & 0.536 & $-0.32_{-0.20}^{+0.20}$ & $45.33_{-2.82}^{+3.05}$ & $91.248 / 76$ & $4.47_{-0.61}^{+0.68}$ & $15.21_{-1.36}^{+1.61}$ & $0.57_{-0.09}^{+0.09}$ & $1.15_{-0.11}^{+0.10}$ & $11.08_{-4.18}^{+7.40}$ & $0.17_{-0.06}^{+0.09}$ & $90.915 / 75$ & $2.83_{-0.15}^{+0.15}$ & $0.49_{-0.05}^{+0.04}$ & 0.9868 \\
\hline 36 & 05:49:46.105 & 0.200 & $-1.08_{-0.44}^{+0.49}$ & $24.37_{-6.21}^{+4.45}$ & $41.745 / 27$ & $1.91_{-0.43}^{+0.57}$ & $9.01_{-0.86}^{+1.00}$ & $1.13_{-0.41}^{+1.01}$ & $1.10_{-0.12}^{+0.11}$ & $666.35_{-505.86}^{+2782.43}$ & $1.30_{-0.48}^{+0.67}$ & $38.411 / 26$ & $0.87_{-0.12}^{+0.05}$ & $0.11_{-0.09}^{+0.00}$ & 0.9913 \\
\hline 37 & 06:06:41.596 & 1.000 & $0.17_{-0.19}^{+0.19}$ & $25.49_{-1.65}^{+1.76}$ & $82.334 / 69$ & $3.84_{-0.59}^{+0.57}$ & $12.00_{-2.14}^{+2.89}$ & $0.75_{-0.12}^{+0.10}$ & $0.63_{-0.11}^{+0.12}$ & $26.46_{-9.90}^{+20.71}$ & $0.24_{-0.15}^{+0.36}$ & $70.145 / 68$ & $3.79_{-0.22}^{+0.18}$ & $0.87_{-0.07}^{+0.04}$ & 0.9990 \\
\hline 38 & 07:26:28.960 & 1.672 & $-0.56_{-0.10}^{+0.10}$ & $42.91_{-1.31}^{+1.36}$ & $317.180 / 180$ & $4.23_{-0.17}^{+0.18}$ & $16.19_{-0.55}^{+0.58}$ & $1.07_{-0.04}^{+0.04}$ & $1.70_{-0.06}^{+0.06}$ & $25.99_{-3.72}^{+4.42}$ & $0.19_{-0.03}^{+0.03}$ & $180.111 / 179$ & $13.85_{-0.32}^{+0.28}$ & $1.88_{-0.08}^{+0.08}$ & 1.0000 \\
\hline 39 & $10: 49: 29.944$ & 0.456 & $-1.25_{-0.21}^{+0.22}$ & $59.82_{-7.68}^{+10.20}$ & $53.961 / 50$ & $3.90_{-0.36}^{+0.39}$ & $20.38_{-1.67}^{+1.83}$ & $0.78_{-0.07}^{+0.07}$ & $1.28_{-0.11}^{+0.11}$ & $26.27_{-8.64}^{+13.39}$ & $0.06_{-0.02}^{+0.02}$ & $37.806 / 49$ & $2.76_{-0.16}^{+0.16}$ & $0.31_{-0.04}^{+0.03}$ & 0.9998 \\
\hline 40 & $10: 52: 11.888$ & 0.248 & $-0.51_{-0.46}^{+0.48}$ & $37.71_{-6.56}^{+7.77}$ & $9.511 / 16$ & $3.99_{-1.53}^{+1.28}$ & $14.26_{-3.66}^{+4.56}$ & $0.38_{-0.13}^{+0.12}$ & $0.57_{-0.15}^{+0.14}$ & $11.66_{-7.26}^{+61.30}$ & $0.11_{-0.08}^{+0.18}$ & $10.539 / 15$ & $0.69_{-0.11}^{+0.09}$ & $0.20_{-0.07}^{+0.01}$ & 0.2544 \\
\hline 41 & 19:29:41.189 & 0.432 & $-0.54_{-0.12}^{+0.12}$ & $54.94_{-1.68}^{+1.73}$ & $204.166 / 160$ & $4.19_{-0.34}^{+0.37}$ & $16.85_{-0.55}^{+0.58}$ & $1.07_{-0.07}^{+0.07}$ & $2.86_{-0.10}^{+0.10}$ & $26.87_{-7.64}^{+11.23}$ & $0.28_{-0.04}^{+0.04}$ & $189.646 / 159$ & $5.23_{-0.13}^{+0.13}$ & $0.32_{-0.03}^{+0.03}$ & 1.0000 \\
\hline 42 & $19: 29: 42.941$ & 0.120 & $-0.06_{-0.39}^{+0.44}$ & $66.48_{-5.20}^{+6.06}$ & $27.940 / 33$ & $5.42_{-1.51}^{+1.76}$ & $19.29_{-1.68}^{+2.09}$ & $0.45_{-0.13}^{+0.16}$ & $1.75_{-0.20}^{+0.18}$ & $4.05_{-2.49}^{+9.94}$ & $0.10_{-0.04}^{+0.05}$ & $24.662 / 32$ & $0.85_{-0.06}^{+0.05}$ & $0.12_{-0.04}^{+0.01}$ & 0.9313 \\
\hline
\end{tabular}

\section{Notes.}

a Events 1-40 were happened on 2009 January 22, while bursts 41 and 42 were on 2009 January 30.

b The luminosity of blackbody components in units of $10^{39} \mathrm{erg} \mathrm{s}^{-1}$.

${ }^{c}$ Burst energy fluence in the GBM band (8-200 keV) in units of $10^{-7} \mathrm{erg} \mathrm{cm}^{-2}$.

d Burst energy fluence in the XRT band $(0.5-10 \mathrm{keV})$ in units of $10^{-7} \mathrm{erg} \mathrm{cm}^{-2}$. 


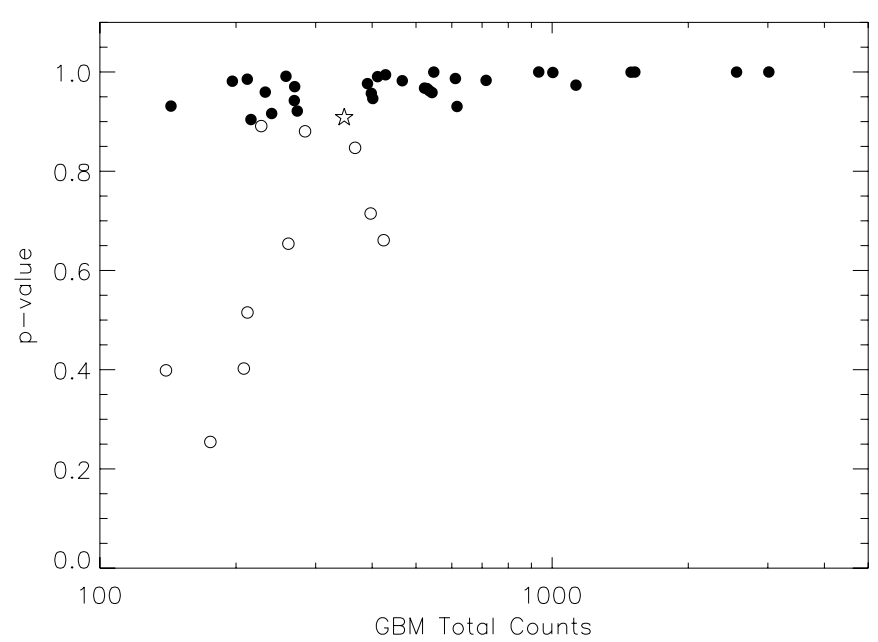

Figure 3. Plot of the $p$-value vs. the total counts in the GBM energy band $(8-200 \mathrm{keV})$. The black dots are the $\mathrm{BB}+\mathrm{BB}$ bursts, the open circles are the intermediate group bursts, and the five-point star is the COMPT burst.

We conclude that the seed model provides a significantly better fit than the other model if $p>0.9$. The $p$-values of all bursts are listed in the last column of Table 2.

We then grouped the 42 bursts into three categories based on their resulting $p$-values: the $\mathrm{BB}+\mathrm{BB}$ burst, where $\mathrm{BB}+\mathrm{BB}$ is significantly preferred to the COMPT model; the COMPT burst, where the COMPT model is significantly better than $\mathrm{BB}+\mathrm{BB}$; and the intermediate group containing bursts for which both the $\mathrm{BB}+\mathrm{BB}$ and COMPT models provide equally acceptable fit results $(p<0.9)$. We find that 31 events are the $\mathrm{BB}+\mathrm{BB}$ bursts, only one is a COMPT burst (event 18), and 10 are in the intermediate group. Figure 3 displays the $p$-values of all bursts versus their total GBM counts. Note that a similar trend is obtained if the $p$-values were plotted with their corresponding total XRT counts. We find that the bright bursts prefer the $\mathrm{BB}+\mathrm{BB}$ model to the COMPT model. This might be an indication of higher opacity, on average, in the more luminous bursts. We explore both the statistical and the correlative behavior of all model parameters in the next sections.

Our joint spectral fits provided the opportunity to investigate the cross-calibration of the XRT and GBM instruments with the two spectral models. We determined a multiplicative factor between the XRT and GBM detectors for each model. The left panel of Figure 4 shows the behavior of this factor from the $\mathrm{BB}+\mathrm{BB}$ model fits as a function of total counts in the brightest GBM detector. The same plot for the COMPT model fits is presented in the right panel of Figure 4. The instruments are perfectly cross-calibrated if the multiplicative factor is equal to 1 (the dotted lines in Figure 4). For brighter bursts, the factor from the $\mathrm{BB}+\mathrm{BB}$ model fit is better constrained, and it does not change significantly from burst to burst: its weighted mean value and $1 \sigma$ error are $1.17 \pm 0.05$. However, the weighted mean of the COMPT model derived factor is $0.58 \pm 0.03$, much smaller and further from 1 . We note that the values obtained from a better fit model are closer to the perfect cross-calibration factor of 1 . We find that the constant factor is not significantly correlated with any spectral parameters. We conclude that the cross-calibration of the XRT and GBM instruments works reasonably well within the fluence and energy range of the bursts in our sample. Future efforts to better understand the cross-calibration of these two instruments should include bursts with wider energy and fluence ranges, and perhaps, different spectral models.

\subsection{Comptonized Model}

We present the distribution of power-law indices obtained from the joint fit of the XRT and GBM spectra (histograms with thick lines) in the left panel of Figure 5. We also present in the same figure the distribution of the indices as obtained by fitting the GBM spectra only (histograms with thin lines). It is clear that the latter fits yield, on average, lower power-law indices (i.e., the spectrum is harder), reflecting the overall broadband curvature of the burst spectra. We fit each distribution with a normal function and find that the joint broadband fit mean index value is $-0.58 \pm 0.09$ (width of $0.43 \pm 0.11$, dotted lines in Figure 5, left panel), while the GBM only fit mean index is $-0.87 \pm 0.05$ (width of $0.42 \pm 0.06$, dashed lines in Figure 5, left panel). We show the distribution of the $E_{\text {peak }}$ values obtained from the joint fit in the right panel of Figure 5. A normal function fit to this distribution yields a mean of $45.0 \pm 2.1 \mathrm{keV}$ with a width of $10.9 \pm 2.2 \mathrm{keV}$. The $E_{\text {peak }}$ values from joint fit agree with those from GBM data only fit very well, see detailed discussion in Section 4.1.

In Figure 6, we present a plot of the joint fits $E_{\text {peak }}$ values versus the observed fluence/flux of bursts in the $0.5-200 \mathrm{keV}$
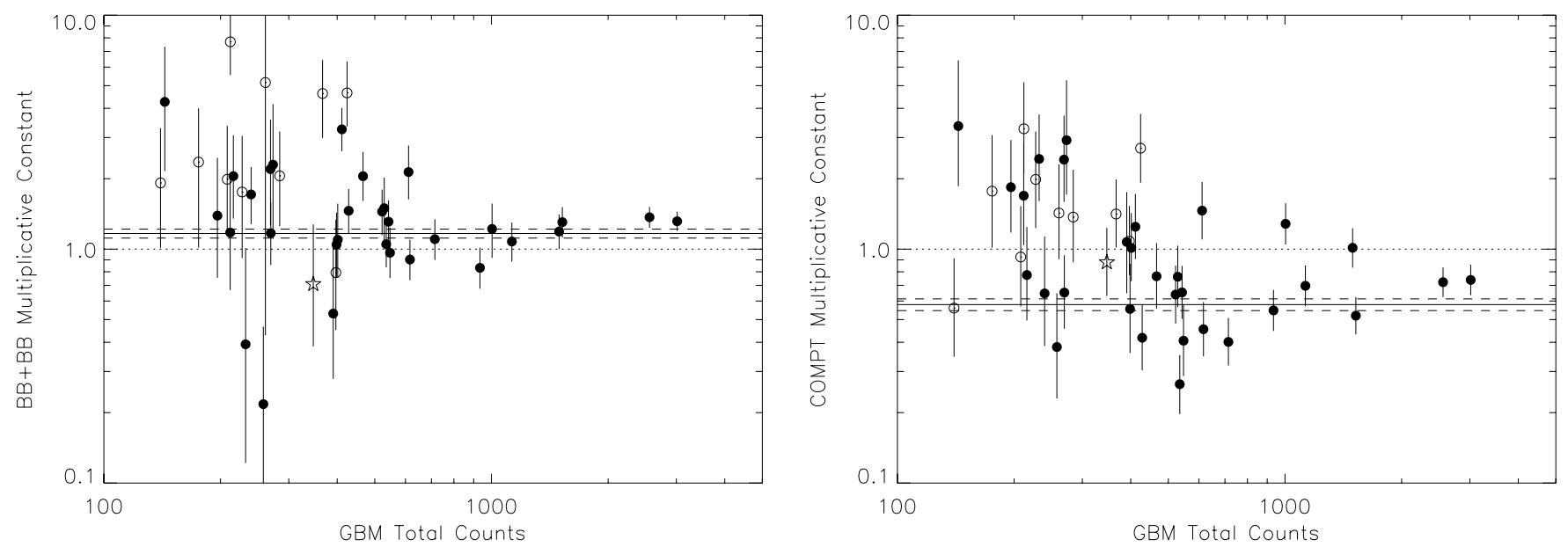

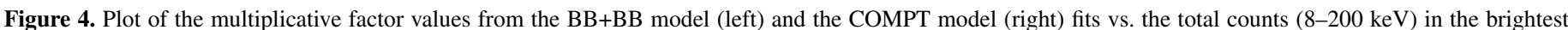

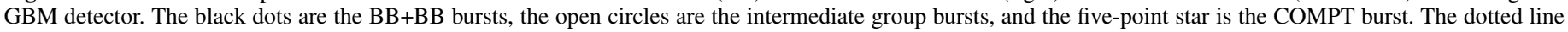
indicates the factor being equal to 1 , and the solid and dashed lines display the weighted average of the factor and its $1 \sigma$ error, respectively. 

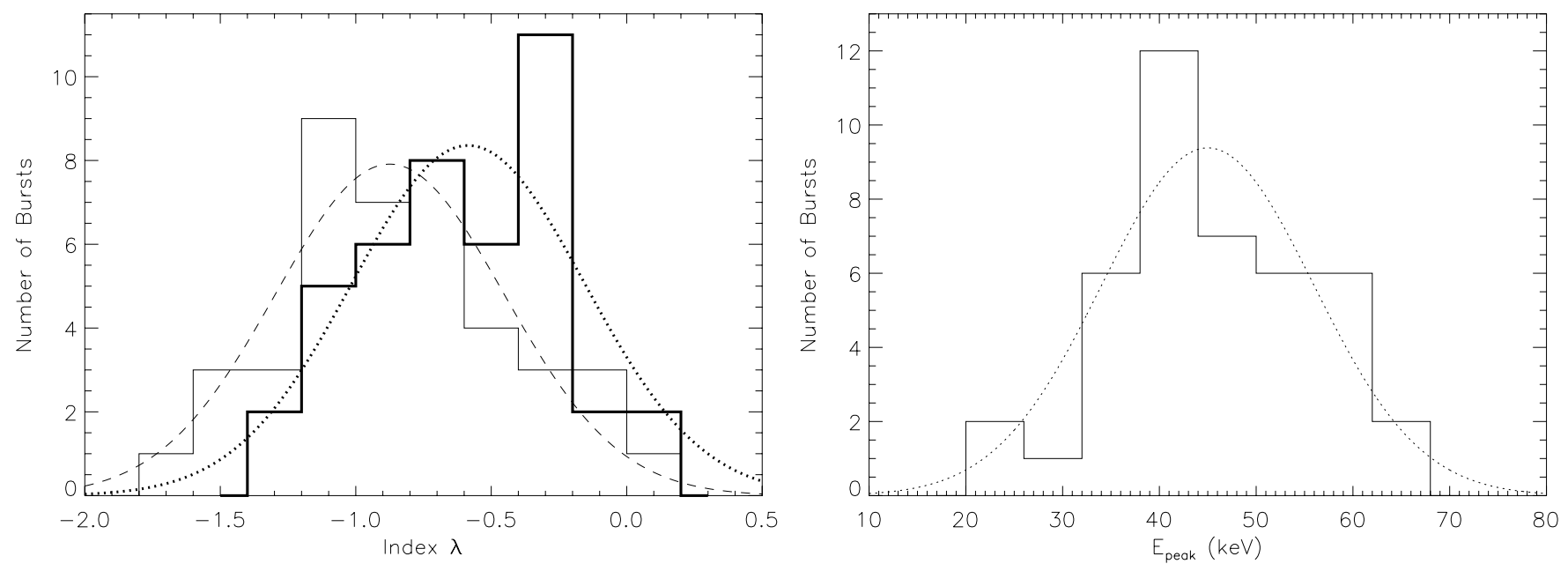

Figure 5. Distributions of the COMPT model index (left) and $E_{\text {peak }}$ (right). The dotted lines show the best-fit normal functions. The thick histograms in the left panel are the index distribution of the XRT-GBM joint fit, while the thinner histograms are the same distribution for the GBM data only fit.
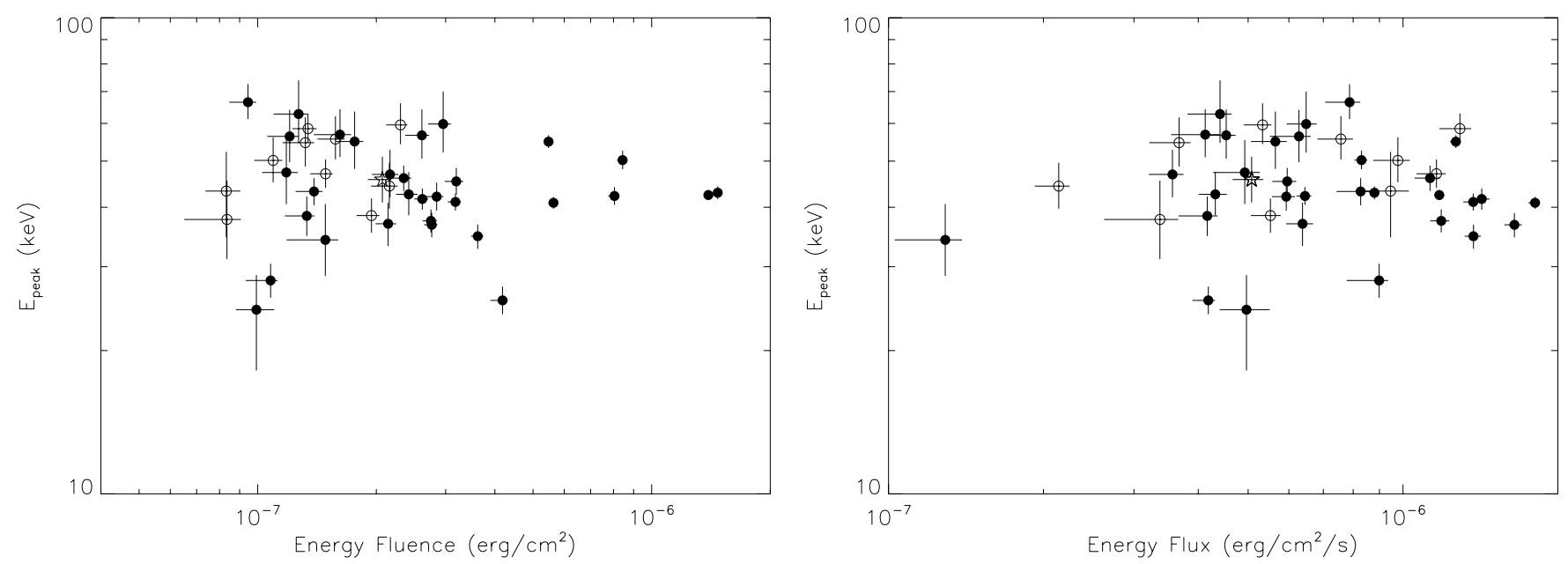

Figure 6. Plot of $E_{\text {peak }}$ vs. observed energy fluence (left) and average flux (right) between 0.5 and $200 \mathrm{keV}$. The black dots are the BB+BB bursts, the open circles are the intermediate group bursts, and the five-point star is the COMPT burst.

range. We do not find any anti-correlation or a broken power-law trend as seen by fitting the GBM data of SGR J1550-5418 (van der Horst et al. 2012) and SGR 0501+4516 (Lin et al. 2011), which we attribute to the fact that the 42 common events cover a much narrower fluence range, about $1 / 3$ of that in the complete GBM burst sample from the same active period (van der Horst et al. 2012).

\subsection{Two Blackbody Model}

From simulation, we find that the $\mathrm{BB}+\mathrm{BB}$ model provides a significantly better fit to 31 common events. We use these $\mathrm{BB}+\mathrm{BB}$ bursts to investigate the properties of the model parameters. In Figure 7, we present the distribution of temperatures of the cool (left panel) and hot (right panel) BB components, respectively. We fit a normal function to these distributions and obtain a mean value for the cool BB temperatures of $4.4 \pm 0.2 \mathrm{keV}$ (width, $0.8 \pm 0.1 \mathrm{keV}$ ), and for the hot $\mathrm{BB}$ temperatures of $16.0 \pm 0.4 \mathrm{keV}$ (width $2.2 \pm 0.4 \mathrm{keV}$ ). We also calculated the weighted mean values for cool and hot BB temperatures, which are $4.2 \pm 0.1 \mathrm{keV}$ and $14.8 \pm 0.2 \mathrm{keV}$, as well as their standard deviations, $0.9 \mathrm{keV}$ and $2.7 \mathrm{keV}$. These mean temperatures are in agreement within uncertainties with the values obtained by fitting only GBM spectra of SGR J1550-5418 bursts (van der Horst et al. 2012) and similar to the BB+BB temperature values obtained for the bursts of other SGR sources (Feroci et al. 2004; Olive et al. 2004; Israel et al. 2008; Lin et al. 2011).

In Figure 8, we demonstrate the anti-correlation between the emission area and the temperature of the cool and hot BB components. The Spearman rank order correlation test yields a correlation coefficient for the hot BB of -0.79 with a chance probability of $1.40 \times 10^{-7}$. The correlation for the cool $\mathrm{BB}$ is not as significant as the hot component: its Spearman correlation coefficient is -0.62 with a probability of $1.75 \times 10^{-4}$. We fit the emission area versus temperature for the cool and hot BB with power laws, and obtain powerlaw indices of $-1.5 \pm 1.4$ and $-4.5 \pm 0.9$, for the cool and hot components, respectively. We also fit the emitting area versus the two BB temperatures together with a single power law and obtain the best-fit power-law index of $-3.5 \pm 0.2$ (shown as the solid line in Figure 8). Note that this value is very close to the theoretical expectation from a single BB with fixed luminosity, $R^{2} \propto(k T)^{-4}$. The observed departure at the high-temperature end from this ideal form reflects the higher luminosity present in the hot $\mathrm{BB}$ component relative to the cooler 

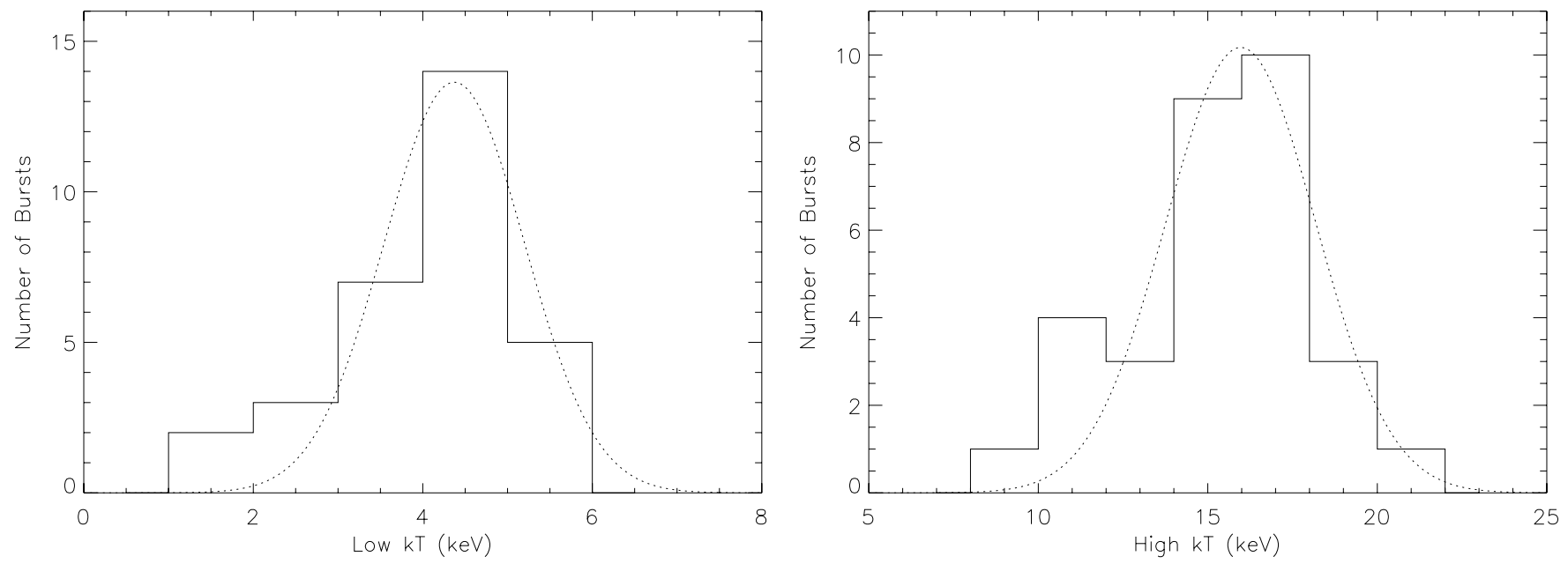

Figure 7. Distributions of the temperatures of the two blackbody components of the BB+BB model. The dotted lines show the best-fit normal functions.

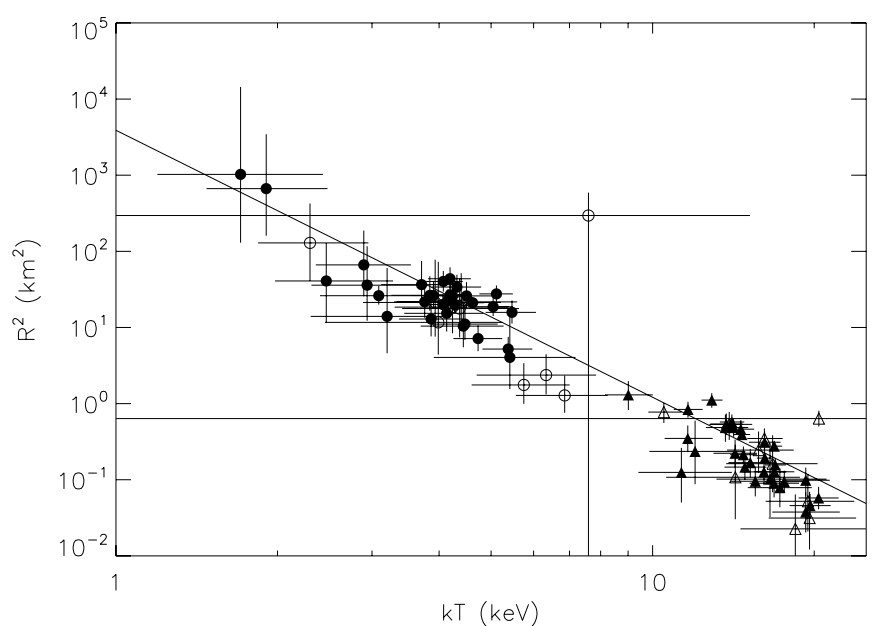

Figure 8. Emission area as a function of blackbody temperature for both blackbody components of the $\mathrm{BB}+\mathrm{BB}$ model fit. The hot and cool blackbody components are displayed with triangles and circles, respectively. The filled symbols are the $\mathrm{BB}+\mathrm{BB}$ bursts. The intermediate group bursts are shown as open symbols. The solid line indicates the $R^{2} \propto(k T)^{-3.5}$ relation, the best-fit power-law function with all emission areas and temperatures for $\mathrm{BB}+\mathrm{BB}$ group bursts.

one; on average, the hot BB energy is about twice the one emitted from the cool BB. The two energies are highly correlated with a Spearman correlation coefficient of 0.88 , corresponding to a chance probability of $6.45 \times 10^{-11}$; a power-law fit yields an index of $0.99 \pm 0.05$ (Figure 9 ).

\section{DISCUSSION}

\subsection{Impact of the XRT Data}

We have used two different instruments, the Swift/XRT and the Fermi/GBM, to perform a time-integrated broadband spectral analysis of 42 common events from SGR J1550-5418. By adding the XRT data, we extended the lower energy bound of our earlier spectral analysis (van der Horst et al. 2012) from $\sim 10 \mathrm{keV}$ to $\sim 0.5 \mathrm{keV}$. For most model parameters, our joint fit results agree well with the results from fitting the GBM spectra only, as shown in Figure 10. The correlation coefficients between all but one (the COMPT power-law index) model parameters derived with and without the XRT data are larger than 0.94, corresponding to a probability smaller than $3.3 \times 10^{-10}$. The

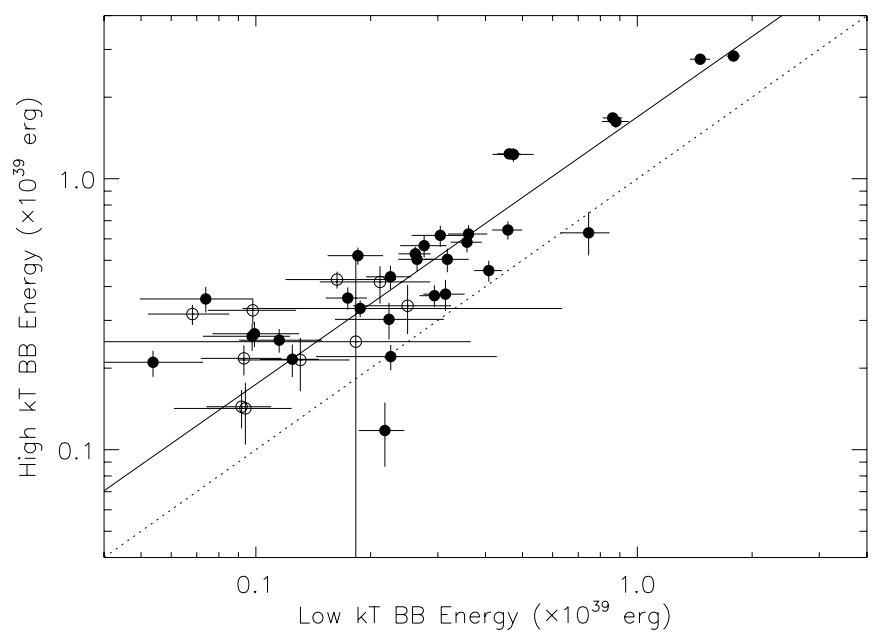

Figure 9. Correlation between the total energy emitted from the hot and cool blackbody components. The filled and open circles present the $\mathrm{BB}+\mathrm{BB}$ and intermediate group bursts, respectively. The best power-law fit $\left(E_{\mathrm{hot}} \propto E_{\mathrm{cool}}^{0.99}\right)$ for the $\mathrm{BB}+\mathrm{BB}$ group is shown as a solid line. The dotted line is where the two $\mathrm{BB}$ components have equal energy.

average COMPT index without the XRT data is $-0.87 \pm 0.05$, consistent within errors with the mean of $\sim-0.92$ obtained from a much larger burst sample (van der Horst et al. 2012). However, the inclusion of the XRT data better constrains the COMPT indices, which become harder than the ones derived from spectral fits to the GBM data alone, as shown in Figure 5. Therefore, we conclude that the COMPT fit to the GBM data only overestimates the emission in the lower energy bands.

Our analysis provides an important diagnostic for the model preference between the COMPT and $\mathrm{BB}+\mathrm{BB}$ models. By adding the XRT data, we find that 31 of 42 bursts are statistically better described by $\mathrm{BB}+\mathrm{BB}$. This fact, combined with the observed steepening of COMPT model indices when excluding XRT data, highlight the generic broad curvature of the SGR J1550-5418 burst spectra. We note here that the joint analysis of XRT and GBM spectra is limited to an absorbed energy fluence range of $8.3 \times 10^{-8}-1.5 \times 10^{-6} \mathrm{erg} \mathrm{cm}^{-2}(0.5-200 \mathrm{keV})$, since the brighter events would cause pile-up in the XRT data and the dimmer events would not yield high enough statistics in the XRT data for a constraining spectral analysis. We discuss the theoretical implications of these results in the next session. 

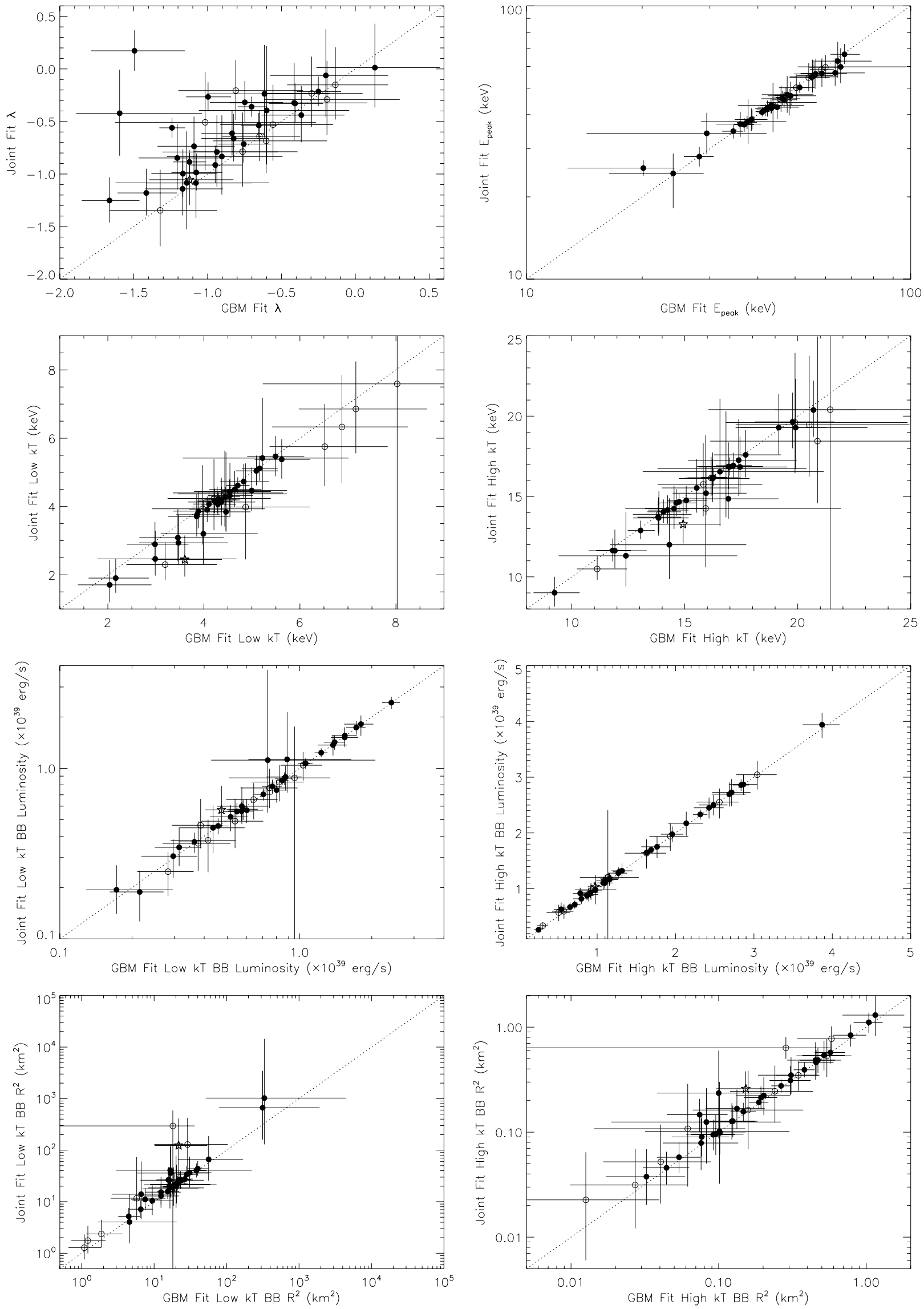

Figure 10. Plots of correlations between model parameters obtained by the joint XRT-GBM fits and the parameters obtained by fitting the GBM data only. The black dots are the BB+BB bursts, the open circles are the intermediate group bursts, and the five-point star is the COMPT burst. The dotted lines represent the $x=y$ trend. 


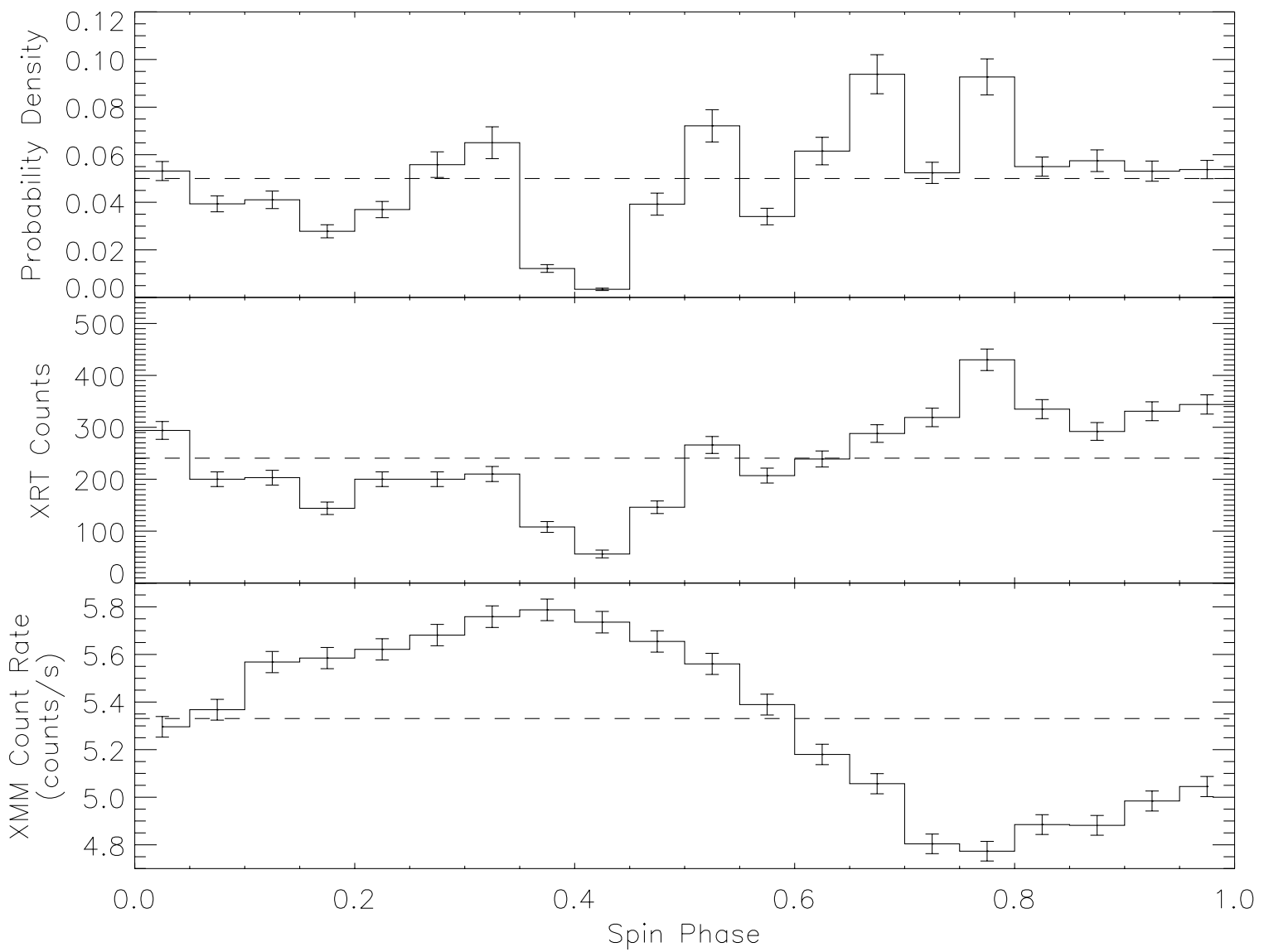

Figure 11. Top: phase probability density profile of the BB+BB group bursts from SGR J1550-5418 ; middle: count phase distribution; bottom: persistent emission pulse profile from contemporaneous $X M M$ observations in the $0.5-10 \mathrm{keV}$ band. The horizontal dashed lines in each panel represent the mean value of the burst phase probability density (top panel), the burst phase counts (middle panel), and the persistent emission count rate (bottom panel).

We also noted the fact that Israel et al. (2008) investigated broadband spectral properties of a very rare event (the storm) from SGR 1900+14, while our investigations are about much more common typical short bursts. Therefore, our results extend the Israel et al. (2008) results to the more common magnetar outbursts.

We investigated here SGR J1550-5418 burst spectra in a time-integrated manner. As seen in the bottom panel of Figure 1, the hardness ratios of the three parts of the burst, designated by the three peaks in the XRT and GBM light curves, show a clear hard to soft spectral evolution. Detailed time-resolved spectral analysis of SGR bursts would provide important insight to the spectral evolution of SGR bursts with time.

\subsection{The BB+BB Model}

The most plausible interpretation of the $\mathrm{BB}+\mathrm{BB}$ model is the emission originating from two hot spots with different temperatures near or on the neutron star surface or in its magnetosphere where local thermodynamic equilibria are achieved. It must be emphasized that a BB spectral fit is an idealization to that emitted by the physical environment of a real photosphere. Due to possible gradients of temperature with optical depth into an evolving region that is approximately in local thermodynamic equilibrium, significant distortions from a true blackbody form are predicted in SGR photospheric spectral models (e.g., Ulmer 1994; Thompson \& Duncan 1995; Lyubarsky 2002; Israel et al. 2008).
To better understand the $\mathrm{BB}+\mathrm{BB}$ behavior and uncover its relation with the spin properties of SGR J1550-5418, we investigated the phase characteristics of the $31 \mathrm{BB}+\mathrm{BB}$ bursts, as follows: We first selected all XRT counts collected during 31 burst intervals and converted their arrival times from the Swift mission time to the corresponding time at the solar system barycenter. We then calculated the spin phase for each burst count using the appropriate spin ephemeris of epoch (MJD) 54854 as reported by Dib et al. (2012) using both RXTE and Swift observations. We present the phase distribution of burst counts in the middle panel of Figure 11. To ensure that the distribution is not dominated by the excessive counts of the brightest bursts, we also calculated the probability density for each phase bin, which is the average of the normalized (by total counts) phase distributions for all bursts, as shown in the top panel of Figure 11. We find that the probability distribution of the burst counts is not uniform over the spin phase of SGR J1550-5418 and the deviation from the mean probability is significant: we calculate the root-mean-square deviation of the phase probability density function from its mean as $0.021 \pm 0.001$. We also compared the phase probability density function to the persistent emission phase profile (bottom panel in Figure 11) obtained using contemporaneous $X M M$ observations (Dib et al. 2012). The phase probability density function is marginally anti-correlated with the persistent emission phase profile in our burst sample with the correlation factor of -0.5 corresponding to a chance probability of $3.4 \times 10^{-2}$. This indicates that the burst emission regions on the neutron star surface are not necessarily associated with the site persistently emitting in X-rays (typically a BB with 
a temperature of $0.5 \mathrm{keV}$ ). This is in agreement with the crustal fracturing mechanism for SGR bursts (Thompson \& Duncan 1995; Braithwaite \& Spruit 2006; Perna \& Pons 2011) as any portion of the solid crust can fracture if the magnetic stress built up is near the threshold to rupture. We also find that the burst probability of some spin phases in SGR J1550-5418 is higher. This could be attributed to a non-uniform surface magnetic field, with some regions having larger magnetic stresses than others.

\subsection{The COMPT Model}

A Comptonization spectrum emerges when low-energy photons are repeatedly upscattered by the thermal electrons in a corona until the photon energy reaches $E \sim k T_{\mathrm{e}}$. We find that the mean value of the Comptonization peak energy, $E_{\text {peak }}$, is $44.8 \mathrm{keV}$, which indicates an average temperature of the thermal electrons, $\left\langle T_{\mathrm{e}}\right\rangle \sim 5.1 \times 10^{8} \mathrm{~K}$. In other words, the average speed of the electrons in the corona is $\sim 0.4 c$, where $c$ is the speed of light.

The essence of Comptonization spectra is discussed at some length in Lin et al. (2011), in the context of GBM observations of SGR J0501+4516 bursts. While the turnover energy provides a diagnostic on the hot electron temperature, the powerlaw slope below the $v F_{v}$ peak energy defines a measure of the opacity in the Comptonizing region. Specifically, in the simplest theoretical constructs (e.g., see Rybicki \& Lightman 1979) the index $\lambda$ for a differential photon spectrum $d N / d E \propto E^{\lambda}$ depends only on the magnetic Compton $y$-parameter $y_{B}=$ $4 k T_{e} /\left(m_{e} c^{2}\right) \max \left\{\tau_{B}, \tau_{B}^{2}\right\}$ via $\lambda=1 / 2-\sqrt{9 / 4+4 / y_{B}}$. Here $\max \left\{\tau, \tau^{2}\right\}$ is the mean number of scatterings per photon by the hot electrons, where $\tau$ is the effective optical depth for scattering, which in our case is modified by the strong magnetic field and thus dubbed the magnetic optical depth and denoted by $\tau_{B}$. Since we require the effective value of $\tau_{B}$, one needs to calculate some Rosseland-type mean opacity, averaged over photon angles and polarizations and accounting for the effects of the strong magnetic fields present, such as anisotropy and polarization-mode switching through scattering in non-uniform $B$ (see also the discussion in Lin et al. 2011). This index is realized only in the energy range somewhat above the soft photon injection energy, $E_{X}$, presumed to be surface thermal $\mathrm{X}$-rays, and somewhat below the characteristic energy associated with the hot thermal electrons, in this case marked via the $v F_{v}$ peak energy $E_{\text {peak }}$. Moreover, the above relationship for $y_{B}$ requires the scattering to be in the Thomson regime, and the mean photon energy to be lower than that of the electrons.

It is evident that $y_{B} \gg 1$ cases yield the flattest Comptonized spectra with index around $\lambda \sim-1$. The COMPT fit indices in Table 2 as well as its distribution in the left panel of Figure 5 are nearly always harder than this, indicating that repeated Compton upscattering has difficulty in generating the observed flat spectra. We note parenthetically that this was also the case for around 1/3-1/2 the bursts reported for SGR J0501+4516 in Lin et al. (2011). The inclusion of XRT data in the current work extends the spectral coverage to much lower energies thus enabling us to determine the value of $\lambda$ significantly better than in previous works. This results in systematically higher values, corresponding to a harder spectral slope, with a mean value of $-0.6 \pm 0.10$ instead of $-0.92 \pm 0.05$, which has made this problem worse. The fact that a large $y_{B}$ is demanded in this fitting protocol would suggest high opacity and strong thermalization might be active in the burst emission region. In such cases, the above dependence of $y_{B}$ on $T_{e}$ and $\tau_{B}$ is not operable, and the Comptonization is saturated, and described instead by a modified Wien or modified BB spectrum (e.g., see Rybicki \& Lightman 1979). Moreover, the upscattered powerlaw photon population is generally substantially lower in total number relative to the seed surface $\mathrm{X}$-ray population (i.e., it presents itself as an X-ray tail), a broadband spectral shape that is at odds with the spectral curvature inferred from the fits here. Hence, there is no strong mandate to prefer a classical, unsaturated Comptonization model for the bursts reported here. Accordingly, the spectra might naturally be expected to exhibit more truly thermalized character, for example, a twoblackbody or multi-blackbody signal emanating from a $\tau_{B} \gg 1$ zone, possibly eliciting spectral distortion imposed by transport within the photosphere (e.g., see Ulmer 1994; Lyubarsky 2002). Perhaps this is what this broadband XRT/GBM analysis has enabled for a magnetar for the first time: the clear discrimination between COMPT and BB+BB spectral models.

L.L. is funded through the Post-Doctoral Research Fellowship of the Turkish Academy of Science. M.G.B. acknowledges support from NASA through grant NNX10AC59A. J.G. is supported by the ERC advanced research grant "GRBs." C.K. is partially funded by the GBM/Magnetar Key Project (NASA grant NNH07ZDA001-GLAST, PI: C. Kouveliotou). A.L.W. acknowledges support from a Netherlands Organization for Scientific Research (NWO) Vidi Grant.

\section{REFERENCES}

Bernardini, F., Israel, G. L., Stella, L., et al. 2011, A\&A, 529, A19 Braithwaite, J., \& Spruit, H. C. 2006, A\&A, 450, 1097

Burrows, D. N., Hill, J. E., Nousek, J. A., et al. 2005, Space Sci. Rev., 120 165

Camilo, F., Ransom, S. M., Halpern, J. P., \& Reynolds, J. 2007, ApJ, 666, L93

Dib, R., Kaspi, V. M., Scholz, P., \& Gavriil, F. P. 2012, ApJ, 748, 3

Duncan, R. C., \& Thompson, C. 1992, ApJ, 392, L9

Enoto, T., Nakazawa, K., Makishima, K., et al. 2010, PASJ, 62, 475

Feroci, M., Caliandro, G. A., Massaro, E., Mereghetti, S., \& Woods, P. M. 2004, ApJ, 612, 408

Gelfand, J. D., \& Gaensler, B. M. 2007, ApJ, 667, 1111

Halpern, J. P., Gotthelf, E. V., Reynolds, J., Ransom, S. M., \& Camilo, F. 2008, ApJ, 676, 1178

Israel, G. L., Esposito, P., Rea, N., et al. 2010, MNRAS, 408, 1387

Israel, G. L., Romano, P., Mangano, V., et al. 2008, ApJ, 685, 1114

Kaneko, Y., Göğüş, E., Kouveliotou, C., et al. 2010, ApJ, 710, 1335

Kouveliotou, C., Dieters, S., Strohmayer, T., et al. 1998, Nature, 393, 235

Kuiper, L., Hermsen, W., den Hartog, P. R., \& Urama, J. O. 2012, ApJ, 748, 133

Lamb, R. C., \& Markert, T. H. 1981, ApJ, 244, 94

Lin, L., Kouveliotou, C., Baring, M. G., et al. 2011, ApJ, 739, 87

Lyubarsky, Y. E. 2002, MNRAS, 332, 199

Lyutikov, M. 2003, MNRAS, 346, 540

Mallozzi, R. S., Preece, R. D., \& Briggs, M. S. 2008, RMFIT: A Light Curve and Spectral Analysis Tool (Huntsville, AL: Univ. Alabama)

Meegan, C., Lichti, G., Bhat, P. N., et al. 2009, ApJ, 702, 791

Mereghetti, S. 2008, A\&AR, 15, 225

Mereghetti, S., Götz, D., Weidenspointner, G., et al. 2009, ApJ, 696, L74

Ng, C.-Y., Kaspi, V. M., Dib, R., et al. 2011, ApJ, 729, 131

Olive, J.-F., Hurley, K., Sakamoto, T., et al. 2004, ApJ, 616, 1148

Perna, R., \& Pons, J. A. 2011, ApJ, 727, L51

Rybicki, G. B., \& Lightman, A. P. 1979, Radiative Processes in Astrophysics (New York: Wiley), 393

Savchenko, V., Neronov, A., Beckmann, V., Produit, N., \& Walter, R. 2010, A\&A, 510, A77

Scholz, P., \& Kaspi, V. M. 2011, ApJ, 739, 94

Thompson, C., \& Duncan, R. C. 1995, MNRAS, 275, 255

Ulmer, A. 1994, ApJ, 437, L111

van der Horst, A. J., Kouveliotou, C., Gorgone, N. M., et al. 2012, ApJ, 749, 122

von Kienlin, A., Gruber, D., Kouveliotou, C., et al. 2012, arXiv:1206.4915

Woods, P. M., \& Thompson, C. 2006, in Compact Stellar X-ray Sources, ed. W. H. G. Lewin \& M. van der Klis (Cambridge Astrophysics Series, Vol. 39; Cambridge: Cambridge Univ. Press), 547 